\title{
Fertilidade e longevidade de Spodoptera frugiperda (J. E. Smith) (Lepidoptera: Noctuidae) em genótipos de milho
}

\author{
Fertility and longevity of Spodoptera frugiperda (J. E. Smith) (Lepidoptera: Noctuidae) \\ in corn genotypes
}

\author{
Letícia Machado dos Santos ${ }^{1}$ Luiza Rodrigues Redaelli* \\ Lúcia Maria Guedes Diefenbach ${ }^{3}$ Caio Fábio Stoffel Efrom $^{4}$
}

\section{RESUMO}

Spodoptera frugiperda (J. E. SMITH) é uma das principais pragas da cultura do milho, entretanto, estudos a respeito de sua biologia, na cultura do milho doce, são raros. Assim, este trabalho objetivou avaliar o efeito dos genótipos ELISA, BR 400 (milhos doce) e BR PAMPA (milho comum) na fase reprodutiva de $S$. frugiperda no que tange aos períodos de pré-oviposição, oviposição e pós-reprodutivo, número de posturas/fêmea e de ovos/ postura, viabilidade dos ovos, período de incubação, intervalo entre oviposições e longevidade. Em condições de laboratório $\left(25 \pm 1{ }^{\circ} \mathrm{C} ; 70 \pm 10 \% \mathrm{UR}\right.$; fotofase 12 horas $)$, 60 lagartas/genótipo foram alimentadas com folhas dos três genótipos. A partir das pupas, foram formados 15, 20 e 24 casais em ELISA, BR 400 e BR PAMPA, respectivamente. Diariamente, registraram-se os números de posturas/fêmea e de ovos/postura. Não se verificou diferença entre os genótipos ELISA, BR 400 e BR PAMPA, respectivamente, em relação a: período de pré-oviposição $(4,78 ; 3,94 ; 3,94$ dias $)$; oviposição $(7,55 ; 6,5 ; 6,11$ dias $)$ e pós-reprodutivo $(4,87 ; 4,64 ; 5,5$ dias $) ;$ número de posturas/fêmea $(6,33 ; 5,25 ; 4,88)$ e de ovos/postura $(216,72 ; 215,03 ; 226,02)$; viabilidade dos ovos $(59,02$; $69,76 ; 60,81 \%)$; intervalo entre oviposiçôes $(0,63 ; 0,86$; 1,0 dias); longevidade dos machos (15,22;13,62;13,29 dias) e fêmeas $(16,33 ; 14,06 ; 15,65$ dias) com prole; $e$ dos machos $(19,17 ; 13,67 ; 13,86$ dias) e fêmeas $(16,43$; 8,$25 ; 19,37$ dias) sem prole. O período de incubação dos ovos das fêmeas oriundas de lagartas mantidas em $B R$ 400 foi mais longo do que o dos demais, sendo a diferença estatisticamente significativa.

Palavras-chave: lagarta-do-cartucho-do-milho, milho doce, oviposição, fecundidade.

\begin{abstract}
Spodoptera frugiperda (J. E. SMITH) is one of the main corn crop pest. Neverthless, studies about its biology in the sweet corn crop are scarce. This study aimed to evaluate the effect of the genotypes ELISA, BR 400 (sweet corn) and BR PAMPA (field corn) in the reproductive phase of $\boldsymbol{S}$. frugiperda concerning: preoviposition, oviposition and post-reproduction periods; number of ovipositions/female and eggs/oviposition; eggs viability; incubation period; interval between ovipositions and longevity. In laboratory conditions (25 $\pm 1{ }^{\circ} \mathrm{C} ; 70 \pm 10 \% \mathrm{RH} ; 12$ hours photophase), 60 larval genotype were fed with leaves of three genotypes. From the pupae, 15, 20 and 24 couples were formed in ELISA, $B R 400$ and BR PAMPA, respectively. The number of ovipositions per female and eggs per oviposition was daily registered. Differences between the genotypes ELISA, BR 400 and BR PAMPA, respectively, were not noticed, concerning: pre-oviposition (4.78; 3.94; 3.94 days); oviposition $(7.55 ; 6.5 ; 6.11$ days) and postreproductive $(4.87 ; 4.64 ; 5.5$ days) periods; number of ovipositions/female (6.33; 5.25; 4.88) and eggs/ oviposition $(216.72 ; 215.03 ; 226.02)$; eggs viability $(59.02 ; 69.76 ; 60.81 \%)$; interval between ovipositions (0.63; 0.86; 1.0 days); longevity of male (15.22; 13.62; 13.29 days) and female $(16.33 ; 14.06 ; 15.65$ days) with offspring and of the male (19.17; 13.67; 13.86 days) and female $(16.43 ; 8.25 ; 19.37$ days) without offspring. The incubation period of the eggs generated by females whose caterpillars were kept in BR 400 genotype was numerically longer than the others.
\end{abstract}

Key words: fall armyworm, sweet corn, oviposition, fecundity.

\footnotetext{
'Engenheiro Agrônomo, MSc, Fitotecnia, Programa de Pós-graduação (PPG) em Fitotecnia, Faculdade de Agronomia, Universidade Federal do Rio Grande do Sul (UFRGS).

${ }^{2}$ Engenheiro Agrônomo, Doutor, Professor Adjunto, Departamento de Fitossanidade, Faculdade de Agronomia, UFRGS. Av. Bento Gonçalves, 7712, 91540-000, Porto Alegre, RS. Autor para correspondência. E- mail: luredael@vortex.ufrgs.br ${ }^{3}$ Biólogo, Doutor, Professor Colaborador, PPG Fitotecnia, Faculdade de Agronomia, UFRGS.

${ }^{4}$ Estudante de graduação do curso de Agronomia, Faculdade de Agronomia, UFRGS.
} 


\section{INTRODUÇÃO}

Spodoptera frugiperda (J. E. SMITH), embora originária das zonas tropicais e subtropicais das Américas (LUGINBILL, 1928), também pode se distribuir, amplamente, pelas zonas temperadas do continente norte-americano, durante os períodos de primavera e de verão.

Apesar de tratar-se de uma espécie polífaga, no Brasil, destaca-se como uma das principais pragas da cultura do milho, sendo comumente referida como lagarta-do-cartucho-do-milho. Sua importância devese não somente aos danos provocados, mas especialmente à dificuldade de seu controle (LEIDERMAN \& SAUER, 1953; CRUZ \& TURPIN, 1983; GASSEN, 1996). Na cultura do milho, o ataque pode ocorrer desde a fase de plântula até o pendoamento e espigamento, quando propicia a entrada de patógenos e umidade, determinando o apodrecimento das espigas (ÁVILA et al., 1997). A capacidade de danos da lagarta é influenciada pelo vigor da planta e pelo clima. Na região tropical, os danos podem ser severos, com até $60 \%$ de redução no rendimento de grãos (GASSEN, 1996). Em se tratando de genótipos de milho doce, além dos danos anteriormente referidos, ocorre a depreciação comercial do produto, tendo em vista que o produto final é consumido "in natura".

Estudos relacionados à fase reprodutiva de insetos-praga são importantes, uma vez que fornecem subsídios para a compreensão de aspectos como o potencial de dano e a dinâmica populacional, bem como a taxa de crescimento, a flutuação e a distribuição espacial, permitindo assim o estabelecimento de métodos para o controle.

O potencial reprodutivo, o comportamento, a fecundidade e a fertilidade de $S$. frugiperda têm sido estudados sob uma variedade de condições ambientais, controladas ou não, e sob uma diversidade de dietas artificiais e naturais. Particularmente, a respeito da fecundidade, existem vários estudos (LUGINBILL, 1928; BURTON \& PERKINS, 1972; SIMMONS \& MARTI Jr., 1992; ROGERS \& MARTI Jr., 1994a).

Este trabalho teve como objetivo avaliar o efeito de diferentes genótipos de milho (doce e comum), quanto aos aspectos reprodutivos dos adultos de $\boldsymbol{S}$. frugiperda, tais como: períodos de pré-oviposição, de oviposição e pós-reprodutivo, número de posturas/fêmea e de ovos/postura, viabilidade dos ovos, período de incubação, intervalo entre oviposições e longevidade.

\section{MATERIAL E MÉTODOS}

Lagartas neonatas obtidas a partir de posturas provenientes de uma criação massal, foram individualizadas em caixas plásticas transparentes $(11 \mathrm{~cm} \times 11 \mathrm{~cm} \times 3,5 \mathrm{~cm})$. Em condições de laboratório $\left(25 \pm 1^{\circ} \mathrm{C} ; 70 \pm 10 \% \mathrm{UR}\right.$; fotofase 12 horas), foram alimentadas, sem restrição, durante toda a fase larval, com seções de folhas de plantas, com 25 a 40 dias de idade, de três genótipos de milho: ELISA, BR 400 (milhos doce) e BR PAMPA (milho comum). Foram utilizadas 60 lagartas por genótipo. As plantas de milho foram cultivadas em parcelas, na área experimental do Departamento de Fitossanidade, UFRGS, Porto Alegre $\left(30^{\circ} 01^{\prime} \mathrm{S}\right.$ e $\left.50^{\circ} 13^{\prime} \mathrm{W}\right), \mathrm{RS}$, no período de outubro a novembro de 2000.

As pupas obtidas foram sexadas pela técnica de BUTT \& CANTU (1962). Assim, formaramse 15,20 e 24 casais, nos genótipos ELISA, BR 400 e BR PAMPA, respectivamente. Os casais foram mantidos nas mesmas condições descritas anteriormente, em gaiolas cilíndricas de tubos de PVC ( $12 \mathrm{~cm}$ de diâmetro x $20 \mathrm{~cm}$ de altura), fechadas numa extremidade com placa de Petri e na outra com tecido tipo filó e revestidas internamente com papel jornal, utilizado como substrato para as posturas. Os casais foram alimentados até a morte com uma solução aquosa de mel a $10 \%$, trocada a cada dois dias.

As gaiolas eram revisadas diariamente, quando também era anotado o número de posturas efetuadas por fêmea e de ovos por postura. Cada postura foi individualizada em placa de Petri de $6 \mathrm{~cm}$ de diâmetro x $1 \mathrm{~cm}$ de altura, e observada durante cinco dias para se determinar a viabilidade dos ovos. Foram computados os períodos de pré-oviposição, de oviposição e pós-reprodutivo, período de incubação, o intervalo entre oviposições e a longevidade. Também avaliou-se o padrão temporal de distribuição dos ovos em relação à idade das fêmeas.

O delineamento experimental utilizado foi o completamente casualizado, com 15, 20 e 24 repetições (número de casais), respectivamente nos genótipos ELISA, BR 400 e BR PAMPA. Os dados foram submetidos à análise de variância e as médias comparadas pelo teste de Tukey ao nível de 5\% de significância.

\section{RESULTADOS E DISCUSSÃO}

Dos 42 casais acompanhados para se avaliar a fase reprodutiva de S. frugiperda, obteve-se um total de 217 grupos de ovos. As posturas realizadas apresentaram heterogeneidade quanto ao número de 
ovos, variando de 14 a 911 ovos/postura, sendo mais freqüentes, nos genótipos BR 400 e BR PAMPA, grupos com 101 a 200 ovos. No genótipo ELISA, registrou-se que os grupos com 0 a 100 e 101 a 200 ovos foram os de maior frequiência. Verificou-se, em todos os genótipos, que algumas fêmeas, embora mantidas com machos, não apresentaram comportamento de oviposição.

Os períodos de pré-oviposição e de oviposição não apresentaram diferença significativa entre os genótipos avaliados (Tabela 1). O período de pré-oviposição variou de um dia, nos genótipos BR 400 e BR PAMPA, a 16 dias nos genótipos ELISA e BR 400. KASTEN Jr. et al. (1978) registraram um período médio de pré-oviposição de 2,8 dias, para adultos de S. frugiperda, provenientes de lagartas mantidas em folhas de milho, em condições controladas $\left(27 \pm 2^{\circ} \mathrm{C}\right.$; $70 \pm 10 \%$ UR; fotofase $12 \mathrm{~h}$ ). CLAVIJO et al. (1991) observaram um valor médio de 6,1 dias para fêmeas de S. frugiperda provenientes de lagartas mantidas em dieta artificial e condições controladas $\left(25 \pm 1^{\circ} \mathrm{C}\right.$; 70$90 \%$ UR). A diferença nos valores para este período, encontrada no presente estudo, comparando-se à literatura, pode ser atribuída ao efeito dos genótipos e/ou à variação das condições de criação, especialmente da temperatura.

Em relação ao período de oviposição, registrou-se uma variação de dois a 14 , um a 18 e dois a 11 dias, nos genótipos ELISA, BR 400 e BR PAMPA, respectivamente, não havendo diferença significativa entre os mesmos (Tabela 1). O período médio de oviposição correspondeu aproximadamente a $40 \%$ da longevidade média das fêmeas, em todos os genótipos avaliados.

Os intervalos entre as oviposições variaram de 0 a 4,0 a 5 e 0 a 4 dias, respectivamente, para os genótipos ELISA, BR 400 e BR PAMPA, não havendo diferença significativa (Tabela 1).

Em relação ao número de posturas por fêmea e de ovos por postura, não se registrou diferença significativa entre os genótipos (Tabela 1). O número de posturas por fêmeas variou de três a 11, um a $11 \mathrm{e}$ dois a oito, e o número de ovos por postura variou de 23 a 911,26 a 675 e 14 a 901 ovos, respectivamente, em ELISA, BR 400 e BR PAMPA. MELO \& SILVA (1987) constataram para adultos provenientes de lagartas de S. frugiperda, mantidas na cultivar de milho AG 28, um número médio de posturas por fêmea superior $(8,25)$, enquanto com a cultivar AG 64 os autores registraram um número mais próximo ao do presente estudo $(4,45)$.

A viabilidade média dos ovos também não diferiu significativamente entre os tratamentos (Tabela 1), observando-se desde posturas sem viabilidade (em que não ocorreu eclosão de nenhuma lagarta) até posturas totalmente viáveis, sendo que a maior parte das posturas em que os ovos eram inférteis, correspondeu às últimas oviposições efetuadas pelas fêmeas. Os valores médios aqui encontrados foram baixos quando comparados aos registrados na literatura, tanto para $\boldsymbol{S}$. frugiperda quanto para $\boldsymbol{S}$. exigua. MELO \& SILVA (1987) encontraram 94,7\%, $87,66 \%$ e 77,37\%, nas cultivares de milho AG 28, P 6872 e AG 64, respectivamente. KASTEN Jr. et al. (1978)

Tabela 1 - Período médio de pré-oviposição, oviposição, pós-reprodutivo e incubação (dias), número médio de posturas/fêmea, ovos/postura e ovos/fêmea, viabilidade média dos ovos $(\%)$ e intervalo médio entre oviposições (dias) de Spodoptera frugiperda, em condições de laboratório $\left(25 \pm 1^{\circ} \mathrm{C} ; 70 \pm 10 \%\right.$ UR; fotofase 12 horas), em três genótipos de milho. Porto Alegre, RS, 20001, (n= número de casais; $\mathrm{EP}=$ erro padrão; $\mathrm{CV}=$ coeficiente de variação).

\begin{tabular}{|c|c|c|c|c|}
\hline \multirow{3}{*}{ Variáveis } & \multicolumn{2}{|c|}{ Genótipos } & \multirow{2}{*}{\multicolumn{2}{|c|}{ BR PAMPA $(n=17)$}} \\
\hline & \multirow{2}{*}{$\begin{array}{c}\text { ELISA }(n=9) \\
(\bar{x} \pm E P)\end{array}$} & \multirow{2}{*}{$\begin{array}{c}\mathrm{BR} 400(\mathrm{n}=16) \\
(\overline{\mathrm{x}} \pm \mathrm{EP})\end{array}$} & & \\
\hline & & & $(\mathrm{x} \pm \mathrm{EP})$ & $\mathrm{CV}$ \\
\hline Pré-oviposição & $4,78 \pm 1,422$ & $3,94 \pm 0,942$ & $3,94 \pm 0,584$ & 82,60 \\
\hline Oviposição & $7,55 \pm 1,214$ & $6,50 \pm 1,245$ & $6,11 \pm 0,469$ & 56,52 \\
\hline Posturas/fêmea & $6,33 \pm 0,707$ & $5,25 \pm 0,777$ & $4,88 \pm 0,428$ & 45,61 \\
\hline Ovos/postura & $216,72 \pm 20,447$ & $215,03 \pm 17,578$ & $226,02 \pm 19,985$ & 76,34 \\
\hline Ovos/fêmea & $1372,56 \pm 183,459$ & $1141,44 \pm 185,331$ & $1106,82 \pm 142,983$ & 54,81 \\
\hline Viabilidade dos ovos & $59,02 \pm 5,174$ & $69,76 \pm 3,592$ & $60,81 \pm 4,257$ & 57,71 \\
\hline Incubação & $2,23 \pm 0,178 b^{1}$ & $2,75 \pm 0,098 \mathrm{a}$ & $2,28 \pm 0,151 b$ & 49,77 \\
\hline Intervalo entre oviposições & $0,63 \pm 0,169$ & $0,86 \pm 0,191$ & $1,0 \pm 0,171$ & 58,06 \\
\hline Pós-reprodutivo & $4,87 \pm 1,649$ & $4,64 \pm 0,809$ & $5,50 \pm 1,348$ & 85,06 \\
\hline
\end{tabular}

${ }^{1}$ Médias não seguidas da mesma letra, na linha, diferem pelo teste de Tukey a 5\% de probabilidade de erro. 
verificaram para adultos de S. frugiperda, provenientes de lagartas mantidas em folhas de milho, um valor de $87,1 \%$. Assim, os resultados do presente trabalho apontam para uma influência negativa dos genótipos testados na viabilidade das posturas, podendo isso se refletir num menor crescimento populacional e, como conseqüência, menor potencial de dano.

Nos genótipos ELISA, BR 400 e BR PAMPA, o número médio total de ovos depositados por fêmea variou de 440 a 2352, 60 a 2411 e 45 a 2145 ovos, não se verificando diferença significativa entre os mesmos (Tabela 1). Não se observou relação entre a longevidade e o número total de ovos postos por fêmea. Se considerarmos fêmeas de 10 dias de idade, encontramos uma grande variabilidade no número total de ovos postos: 1088, 1504 e 757 ovos, nos genótipos ELISA, BR 400 e BR PAMPA, respectivamente, não se verificando uma relação positiva entre a longevidade e a fecundidade. Para a mesma espécie, MELO \& SILVA (1987) registraram uma média de 1303,18 ovos na cultivar P 6872, bem próxima da encontrada neste trabalho para o genótipo ELISA. ROGERS \& MARTI Jr. (1994b) também constataram um valor médio semelhante ao encontrado no genótipo ELISA, 1372,55 ovos, para fêmeas de $S$. frugiperda, cujas lagartas foram mantidas em uma dieta artificial. Já KASTEN Jr. et al. (1978) registraram um valor médio de 1040,6 ovos, semelhante aos valores encontrados no presente estudo para os genótipos BR 400 e BR PAMPA(Tabela 1). A variabilidade no número de ovos postos por fêmea decorre da quantidade e qualidade de alimento ingerido e/ou fecundidade natural inerente a cada indivíduo (LUGINBILL, 1928). A quantidade e a qualidade do alimento consumido por uma larva afetam a sua performance (taxa de crescimento, tempo de desenvolvimento, peso final, dispersão e sobrevivência) e, em certos casos, afetam também a fecundidade, a fertilidade e a dispersão dos adultos (SCRIBER \& SLANSKY, 1981; BROWNE, 1995). Entretanto, tendo em vista que não se constatou diferença significativa entre os genótipos, em relação ao número de ovos por fêmea, acredita-se que a alimentação da fase larval teve pouco efeito considerando-se esses aspectos.

Quanto ao período médio de incubação, analisando-se um total de 49.432 ovos, verificou-se diferença significativa entre os genótipos (Tabela 1), sendo que o mais longo foi registrado no genótipo BR 400. Os valores observados desta variável foram muito próximos àqueles encontrados por KASTEN Jr. et al. (1978) para adultos de $S$. frugiperda, provenientes de lagartas mantidas em folhas de milho (2,4 dias).
As fêmeas de $\boldsymbol{S}$. frugiperda apresentaram um padrão errático de oviposição, isto é, intervalos irregulares entre as ocasiões de postura e uma grande variação no número de ovos depositados por ocasião de postura. Nas fêmeas mantidas no genótipo ELISA, o maior número de ovos foi posto respectivamente no quarto e quinto dia após a formação do casal (Figura 1A), enquanto que nos genótipos BR 400 e BR PAMPA constatou-se muita variabilidade, sendo os maiores valores encontrados nos períodos do segundo ao sexto dia e do terceiro ao oitavo dia, respectivamente (Figura 1B e C).

Em relação ao período médio pósreprodutivo não se verificou diferença significativa entre os três genótipos avaliados (Tabela 1).

A longevidade média, tanto dos machos quanto das fêmeas com prole, não diferiu significativamente da dos machos e das fêmeas sem prole, entre os genótipos (Tabela 2). Esses resultados sugerem que tanto o investimento na atividade de reprodução quanto o alimento ingerido na fase larval tiveram pouco efeito na longevidade dos indivíduos. MELO \& SILVA (1987), da mesma forma, não observaram efeito diferencial na longevidade de adultos de $\boldsymbol{S}$. frugiperda utilizando três cultivares de milho. Porém, embora não tenha sido observada diferença no grupo que gerou prole, as fêmeas foram mais longevas que os machos, em todos os genótipos. A maior longevidade de fêmeas de $\boldsymbol{S}$. frugiperda também foi constatada nos trabalhos realizados por MELO \& SILVA (1987) e GARCIA \& CLAVIJO (1989).

Nos trabalhos realizados por KASTEN Jr. et al. (1978), MELO \& SILVA (1987) e GARCIA \& CLAVIJO (1989) foram sempre registrados, para a mesma espécie, valores inferiores ao do presente estudo para a longevidade média tanto de fêmeas quanto de machos. Esse aspecto pode ser decorrente das diferentes dietas naturais e artificiais utilizadas e/ ou variações das condições ambientais de criação.

Os genótipos de milho testados não mostraram influência diferencial na fase reprodutiva de S. frugiperda, podendo os genótipos de milho doce gerarem proles semelhantes tanto entre si quanto em comparação com o milho comum. Assim, pode ser esperado um potencial de dano dessa espécie nos genótipos de milho doce testados semelhante àquele encontrado para o milho comum.

\section{CONCLUSÃO}

Em relação à fase reprodutiva, os adultos provenientes dos diferentes genótipos foram semelhantes entre si, exceto no que tange ao período 


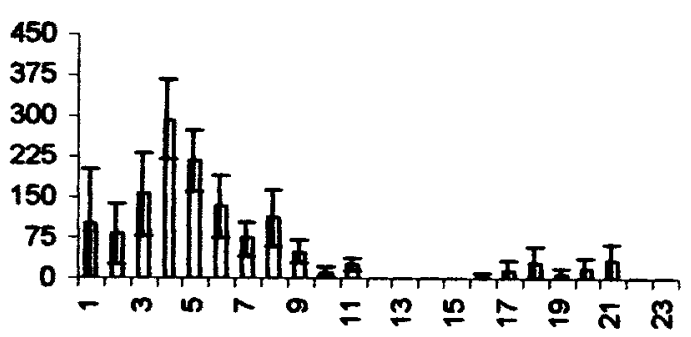

(A)

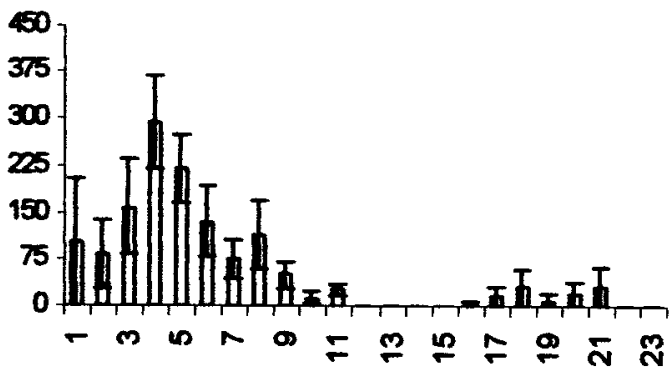

(B)

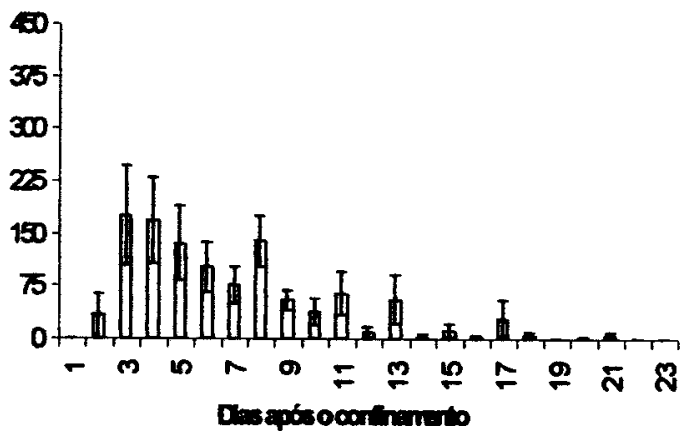

(C)

Figura 1 - Padrão temporal de oviposição de Spodoptera frugiperda nos genótipos ELISA (A), BR 400 (B) e BR PAMPA (C), em condições de laboratório $\left(25 \pm 1^{\circ} \mathrm{C} ; 70 \pm 10 \%\right.$ UR; fotofase 12 horas $)$. Porto Alegre, RS, 2000.

Tabela 2 - Longevidade média de adultos de Spodoptera frugiperda, em dias, em condições de laboratório $\left(25 \pm 1{ }^{\circ} \mathrm{C} ; 70 \pm 10 \%\right.$ UR; fotofase 12 horas), em três genótipos de milho. Porto Alegre, RS, 2000, ( $n=$ número de casais; $\mathrm{EP}=$ erro padrão; $\mathrm{CV}=$ coeficiente de variação).

\begin{tabular}{|c|c|c|c|c|c|c|c|}
\hline & \multicolumn{7}{|c|}{ Genótipo } \\
\hline & \multicolumn{2}{|c|}{ ELISA } & \multicolumn{2}{|c|}{ BR 400} & \multicolumn{3}{|c|}{ BR PAMPA } \\
\hline & $(\overline{\mathrm{x}} \pm \mathrm{EP})$ & $\mathrm{n}$ & $(\mathrm{x} \pm \mathrm{EP})$ & $\mathrm{n}$ & $(\overline{\mathrm{x}} \pm \mathrm{EP})$ & $\mathrm{n}$ & $\mathrm{CV}$ \\
\hline \multicolumn{8}{|c|}{ COM PROLE } \\
\hline Machos & $15,22 \pm 1,622$ & 9 & $13,62 \pm 0,957$ & 16 & $13,29 \pm 1,133 *$ & 17 & 31,89 \\
\hline Fêmeas & $16,33 \pm 1,607$ & & $14,06 \pm 1,283$ & & $15,65 \pm 1,134$ & & 32,16 \\
\hline \multicolumn{8}{|c|}{ SEM PROLE } \\
\hline Machos & $19,17 \pm 2,212$ & 6 & $13,67 \pm 1,054$ & 4 & $13,86 \pm 1,993$ & 7 & 30,10 \\
\hline Fêmeas & $16,43 \pm 3,550$ & & $8,25 \pm 2,323$ & & $19,37 \pm 2,416$ & & 45,85 \\
\hline
\end{tabular}

*Não significativo a 5\% de probabilidade de erro.

Ciência Rural, v. 34, n. 2, mar-abr, 2004. 
de incubação, que em BR 400 foi menor. Estas pequenas diferenças observadas entre os genótipos não resultaram em um número e viabilidade diferencial de descendentes, entretanto, o encurtamento do período de incubação, em BR 400, pode refletir num menor tempo de geração, podendo resultar num maior número de gerações e consequientemente em maiores danos.

\section{REFERÊNCIAS BIBLIOGRÁFICAS}

ÁVILA, C.J.; DEGRANDE, P.E.; GOMEZ, S. A. Insetospragas: reconhecimento, comportamento, danos e controle. In: _____- Milho: informações técnicas. Dourados, EMBRAPA, 1997. p.168-180. (Embrapa, circular técnica 5).

BROWNE, L.B. Ontogenic changes in feeding behavior. In: CHAPMAN, R.F.; DE BOER, G. (Eds). Regulatory mechanisms in insect feeding. New York : Chapman \& Hall, 1995. p.307-342.

BURTON, R.L.; PERKINS, W.D. WSB, a new laboratory diet for the corn earworm and the fall armyworm. Journal of Economic Entomology, Tifton, v.65, n.2, p.385-386, 1972 .

BUTT, B.A.; CANTU, E. Sex determination of lepidopterous pupae. Washington : United States Departament of Agriculture-ARS, 1962. p.33-75.

CLAVIJO, S.A. et al. Influencia de la temperatura sobre el desarrolo de Spodoptera frugiperda (Smith) (Lepidoptera: Noctuidae). Agronomía Tropical, Macaray, v.41, n.5-6, p. 245-256, 1991.

CRUZ, I.; TURPIN, F.T. Yield impact of larval infestations of the fall armyworm (Lepidoptera: Noctuidae) to midwhorl growth stage of corn. Journal of Economic Entomology, Indiana, v.76, n.5, p.1052-1054, 1983.
GARCIA, J.L.; CLAVIJO, S.A. Efecto de la alimentación sobre la longevidad, fertilidad y fecundidad de Spodoptera frugiperda (Smith). Boletín Entomologico Venezuelano, Macaray, v.5, n.3, p.47-53, 1989.

GASSEN, D.N. Manejo de pragas associadas à cultura milho. Passo Fundo : Aldeia Norte, 1996. Cap.5. p.85-104.

KASTEN Jr.P.; PRECETTI, A.A.C.M.; PARRA, J.R.P. Dados biológicos comparativos de Spodoptera frugiperda (J.E. SMITH, 1797) em duas dietas artificiais e substrato natural. Revista de Agricultura, Piracicaba, v.53, p.69$78,1978$.

LEIDERMAN, L.M.; SAUER, H.F.G. A lagarta dos milharais. O Biológico, v.19, p.105-113, 1953.

LUGINBILL, P. The fall armyworm. United States Departament of Agriculture Technical Bulletin, Washington, v.34, p.1-91, 1928.

MELO, M.; SILVA, R.F.P. Influência de três cultivares de milho no desenvolvimento de Spodoptera frugiperda (J.E. Smith, 1797) (Lepidoptera: Noctuidae). Anais da Sociedade Entomológica do Brasil, Porto Alegre, v.16, n.1, p.37-49, 1987.

ROGERS, C.E.; MARTI Jr., O.G. Effects of age at first mating on the reproductive potential of the fall armyworm (Lepidoptera: Noctuidae). Environmental Entomology, Tifton, v.23, n.2, p.322-325, 1994a.

ROGERS, C.E.; MARTI Jr., O.G. Reproductive potential of once-mated moths of the fall armyworm (Lepidoptera: Noctuidae). Florida Entomologist, Tifton, v.77, n.4, p.402410, $1994 \mathrm{~b}$.

SCRIBER, J.M.; SLANSKY Jr., F. The nutritional ecology of immature insects. Annual Review of Entomology, Wisconsin, v.26, p.183-211, 1981.

SIMMONS, A.M.; MARTI Jr., O.G. Mating by the fall armyworm (Lepidoptera: Noctuidae): frequency, duration, and effect of temperature. Environmental Entomology, Tifton, v.21, n.2, p.371-375, 1992. 